\title{
Intestinal barrier regulates immune responses in the liver via IL-10-producing macrophages
}

\author{
Nobuhito Taniki, ${ }^{1}$ Nobuhiro Nakamoto, ${ }^{1}$ Po-Sung Chu, ${ }^{1}$ Yohei Mikami, ${ }^{1}$ Takeru Amiya,,${ }^{1,2}$ \\ Toshiaki Teratani, ${ }^{1}$ Takahiro Suzuki, ${ }^{1}$ Tomoya Tsukimi, ${ }^{3}$ Shinji Fukuda, ${ }^{3,4}$ Akihiro Yamaguchi, ${ }^{1}$ \\ Shunsuke Shiba, ${ }^{1}$ Rei Miyake, ${ }^{1}$ Tadashi Katayama, ${ }^{1}$ Hirotoshi Ebinuma, ${ }^{1}$ and Takanori Kanai ${ }^{1}$ \\ 'Division of Gastroenterology and Hepatology, Department of Internal Medicine, Keio University School of Medicine, \\ Shinanomachi, Tokyo, Japan. ²Research Unit/Frontier Therapeutic Sciences, Sohyaku, Innovative Research Division, \\ Mitsubishi Tanabe Pharma Corporation, Yokohama, Japan. ${ }^{3}$ Institute for Advanced Biosciences, Keio University, Tsuruoka, \\ Yamagata, Japan. ${ }^{4}$ Precursory Research for Embryonic Science and Technology, Japan Science and Technology Agency, \\ Kawaguchi, Saitama, Japan.
}

The gut-liver axis is of clinical importance as a potential therapeutic target in a wide range of liver diseases; however, the mechanisms underlying interactions between microbial products and immune responses in the liver remain unknown. In this study, we demonstrated that IL-10producing macrophages contribute to immune tolerance in the inflamed liver under intestinal barrier disruption in a murine tandem model of dextran sulfate sodium (DSS) colitis and concanavalin A (Con A) hepatitis. Intestinal barrier disruption protected mice from subsequent liver injury, and the severity of colitis directly affected susceptibility to such injury. The protective effect of DSS-Con A was canceled in gut-sterilized mice, suggesting that gut microbiota play a substantial role in this process. Altered gut microbiota and their metabolites, along with a disrupted intestinal barrier, directly gave rise to immunological permissiveness in the inflamed liver. We identified 1-methylnicotinamide (1-MNA) as a candidate metabolite capable of suppressing liver injury with the potential to induce IL-10-producing macrophages. Consistently, expression of nicotinamide $\mathrm{N}$-methyltransferase, which converts nicotinamide to 1-MNA, was upregulated in the liver of DSSCon A mice, and this effect was abrogated by gut sterilization. Collectively, our results provide a mechanistic insight into the regulation of immunological balance in the liver via the gut-liver axis.

Conflict of interest: The authors have declared that no conflict of interest exists.

Submitted: November 28, 2016

Accepted: May 8, 2018

Published: June 21, 2018

\section{Reference information:}

JCI Insight. 2018;3(12):e91980.

https://doi.org/10.1172/jci.

insight. 91980 .

\section{Introduction}

Under physiological conditions, the liver is continuously exposed to gut-derived antigens through the portal vein, and bacterial degradation of metabolites fermented by commensal bacteria reach the liver and influence its innate and adaptive immune responses (1-3). Intestinal inflammation can lead to a leaky gut, and live bacteria and bacterial products migrate from the gastrointestinal tract to portal or systemic circulation following intestinal barrier disruption (4), after which they can activate immune cells in the liver. Liver tolerance is a well-known organ-specific phenomenon possibly caused by its unique anatomical location. That immune responses in the liver trend toward immune permissiveness was first recognized in a porcine orthotopic liver transplantation model (5). The liver is indispensable to the maintenance of oral tolerance against nutrients, gut-derived bacterial metabolites, LPS from the cell walls of gram-negative bacteria, and cellular debris that enters the liver via the portal vein from the intestine. In general, the presentation of antigens by liver antigen-presenting cells promotes initial proliferation, followed by clonal exhaustion or apoptosis of $\mathrm{T}$ cells (6), resulting in the maintenance of immunological balance under homeostatic conditions. Recently, gut microbiota has been reported to both help and overcome liver tolerance and contribute to the pathogenesis of liver diseases (7-10). Clinically, primary sclerosing cholangitis (PSC) is a well-documented example of chronic hepatobiliary disease highly associated with inflammatory bowel disease (IBD) (11), indicating that the gut-liver axis plays a role on the pathogenesis $(7,12)$. These phenomena suggest that increased intestinal permeability with continuous exposure to gut-microbial products might influence immunological responses in the liver. However, the underlying mechanisms by which subsets of immune cells contribute to immunolog- 
ical activation and tolerance in the liver remain unclear.

In this study, we demonstrated that preexisting intestinal barrier damage induced by dextran sulfate sodium (DSS) unexpectedly ameliorated the severity of subsequent immune-mediated acute liver injury models induced by concanavalin A (Con A). Using a tandem model of DSS colitis and Con A hepatitis, we explored the roles of the gut-liver axis in hepatic immune regulation, demonstrating that colitis prior to hepatitis preconditioned hepatic $\mathrm{CD} 11 \mathrm{~b}^{+} \mathrm{F} 4 / 80^{+}$innate immune cells as IL-10-producing regulatory cells in vivo. Furthermore, the amelioration of Con A hepatitis was canceled by gut sterilization, suggesting that immune balance in the liver is potentially regulated by gut microbiota and metabolites.

\section{Results}

Severe mucosal barrier degradation leads to significantly milder liver injury following a sublethal injection of Con A. To clarify whether excessive gut-derived antigen stimulation affects immune responses in the liver, mice were orally administered DSS to induce mucosal barrier degradation, followed by Con A administration to induce acute liver injury. Mice were either untreated or orally administered $2.0 \%$ DSS for 7 days, followed by i.v. PBS or Con A (20 mg/kg) exposure, in distilled water-PBS (DW-PBS), DSS-PBS, DW-Con A, or DSS-Con A experimental groups. DSS-treated mice developed substantial colitis, as demonstrated by body weight loss and disease activity index (DAI) (Figure 1A). Interestingly, DSS-treated mice exhibited significantly milder liver injury, as determined by serology and histology of H\&E staining and TUNEL assay to detect apoptosis, than did untreated mice following a sublethal dose of Con A (Figure 1, B-D). As shown previously, Con A administration upregulated the mRNA expression of proinflammatory cytokines such as TNF- $\alpha$ and IL-6 - in the liver, whereas their expression was significantly decreased in DSS-Con A mice (Figure 1E).

To examine the relationship between the severity of colitis and subsequent liver injury, mice were pretreated with DSS for 3-7 days. We observed that the severity of DSS-induced colitis was correlated with the duration of DSS administration (Figure $1 \mathrm{~F}$ ). Although mice pretreated with DSS for 3 days exhibited more severe liver injury, pretreatment with DSS for 7 days significantly attenuated subsequent liver injury (Figure $1 G)$. Furthermore, mice that recovered from DSS colitis and that had received DW for 3 weeks after DSS treatment developed more severe hepatitis following Con A administration (DSS-DW-Con A) than did DSS-Con A mice (Supplemental Figure 1, A and B; supplemental material available online with this article; https://doi.org/10.1172/jci.insight.91980DS1), suggesting that the severity of colitis directly affected susceptibility to subsequent liver injury in this model. Similarly, 2,4,6-trinitro-benzene sulfonic acid-treated (TNBS-treated) mice, another murine model of colitis, exhibited less susceptibility to Con A-induced acute liver injury. Additionally, DSS-treated mice exhibited less susceptibility to $\alpha$-galactosylceramide-induced $(\alpha$-Galcer-induced) acute liver injury, another murine model of immune-mediated acute liver injury (Supplemental Figure 1, C-F). These results suggested that preexisting colitis with intestinal barrier degradation directly contributed to the attenuation of subsequent liver injury. Additionally, to confirm whether coexisting inflammations compensated for one another, we performed the experiment in the reverse order (i.e., Con A-injected mice were subsequently administered DSS [Supplemental Figure 2A]). We observed that Con A pretreatment did not ablate DSS-induced colitis (Supplemental Figure 2, B and C), suggesting that the unidirectional gut-liver axis contributed to immune tolerance in the liver in this model.

Monocyte-derived macrophages accumulating in the liver following DSS-Con A treatment display decreased TNF$\alpha$ production and increased $I L-10$ production. We then examined the cell types responsible for attenuating liver injury in this model. We found that DSS administration induced an increase in CD11 $\mathrm{b}^{+}$macrophages in the liver, as reported previously (13), and Con A administration also induced a robust increase in CD11 $\mathrm{b}^{+}$macrophages in the liver (Figure 2, A and B). Notably, DSS-Con A-treated mice exhibited the greatest increase in $\mathrm{CD}_{11} \mathrm{~b}^{+}$macrophage number in the liver among the 4 groups (Figure 2, A and B). We then assessed whether DSS-Con A-induced hepatic CD11 b ${ }^{+}$macrophages were ontogenically distinct. As shown in Supplemental Figure 3, A and B, hepatic CD11 b cells from DSS-Con A mice showed a higher expression of conventional macrophage markers F4/80 and lymphocyte antigen 6 complex, locus C1 (Ly6C), and CSF1 receptor (CSF1R), a marker of BM-derived monocytes (14). The expression of C-type lectin-domain family 4 member F (CLEC4F), a marker of resident liver macrophages (Kupffer cells) (15), was unchanged between the 4 groups, and NKp46 (a marker of NK cells) and Ly6G (a marker of neutrophils) were not increased in the DSS-Con A group. Furthermore, DSS-Con A macrophages displayed marked downregulation of major histocompatibility complex class II-IA ${ }^{b}$ expression as compared with that observed in 
A

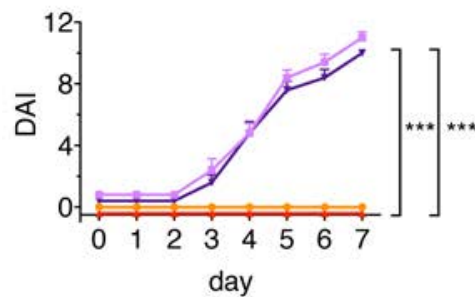

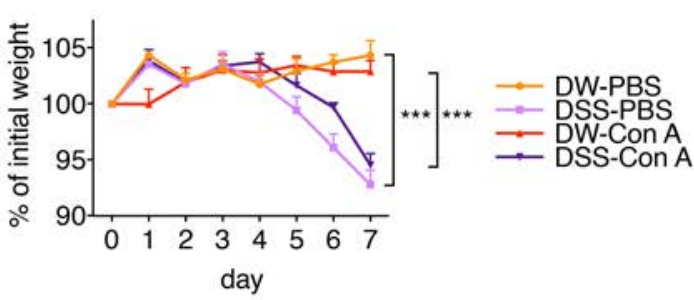

B

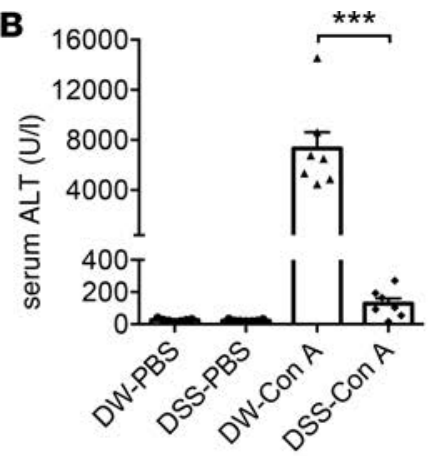

C
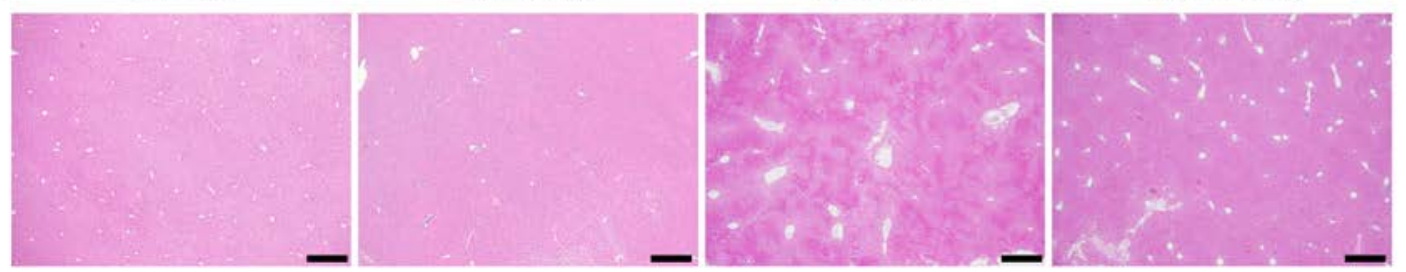

D
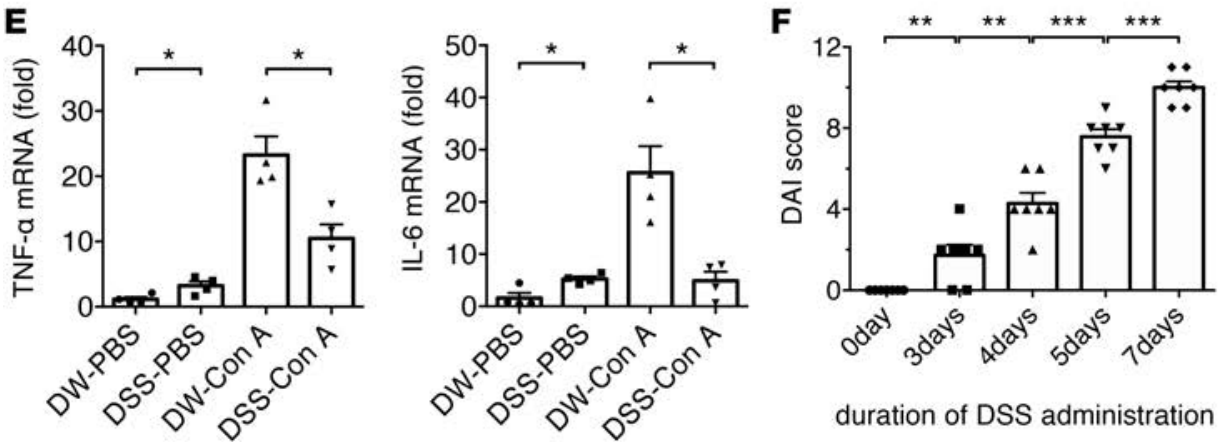

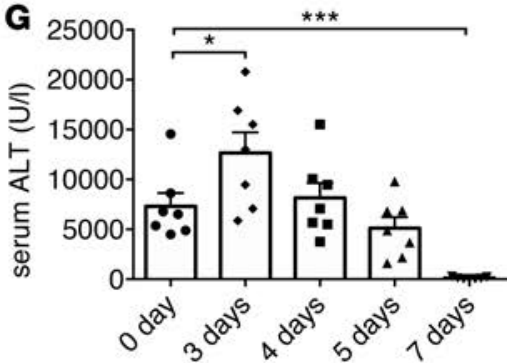

duration of DSS pre-administration

Figure 1. Severe mucosal barrier degradation leads to significantly milder liver injury following a sublethal injection of concanavalin A (Con A). (A) Mice were either untreated or orally administrated $2.0 \%$ dextran sulfate sodium (DSS) followed by intravenous PBS or Con A (20 mg/kg) administration. Mean body weight and disease activity index (DAI) on days 0-7 of each experimental group ( $n=5-7 /$ group). (B) Serum alanine aminotransferase (ALT) level for each experimental group ( $n=5-7 /$ group). (C) Histopathology of H\&E-stained sections of liver. Data are representative of each experimental group. Scale bars: $500 \mu \mathrm{m}$ ( $n=5 /$ group). (D) Representative TUNEL assay images of mouse liver sections from each experimental group ( $n=5 /$ group). Scale bars: $50 \mu \mathrm{m}$. (E) Expression of TNF- $\alpha$ and IL-6 mRNA in whole liver cells ( $n=4 /$ group). (F) DAl for each duration of DSS administration. (C) Serum ALT levels after Con A injection for each duration of DSS pretreatment. Data represent means $\pm \mathrm{SEM}$. ${ }^{*} P<0.05,{ }^{* *} P<0.01,{ }^{* * *} P<0.001$ according to 1-way ANOVA with Tukey's multiple-comparison correction.

DW-Con A macrophages. We previously reported that BM-derived, TNF- $\alpha$-producing C-C chemokine receptor $9^{+} \mathrm{CD} 11 \mathrm{~b}^{+}\left(\mathrm{CCR} 9^{+} \mathrm{CD} 11 \mathrm{~b}^{+}\right)$macrophages play a critical role in Con A-induced acute liver injury by inducing Th1 responses $(16,17)$. Consistently, CCR9 expression in DW-Con A macrophages was significantly elevated, whereas DSS-Con A macrophages lacked CCR9 expression. Unlike endotoxin-tolerant mice (18), upregulation of IL-receptor-associated kinase 3 (IRAK3) was not observed in macrophages derived from the livers of DSS-Con A mice (Supplemental Figure 3C). To examine potential inflammatory cytokine production, liver mononuclear cells (MCs) and colon lamina propria MCs (LPMCs) were stimulated with LPS for 4 hours in vitro, followed by intracellular TNF- $\alpha$ staining. As shown in Figure 2, C and $\mathrm{D}$, hepatic CD11b $\mathrm{b}^{+}$cells derived from DSS-Con A mice produced less TNF- $\alpha$ than did DW-Con A mice, 
A Liver MC

DW-PBS

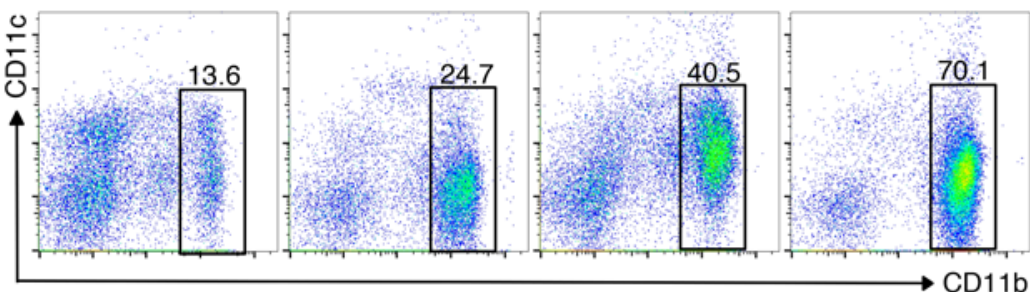

C Liver MC

DW-PBS

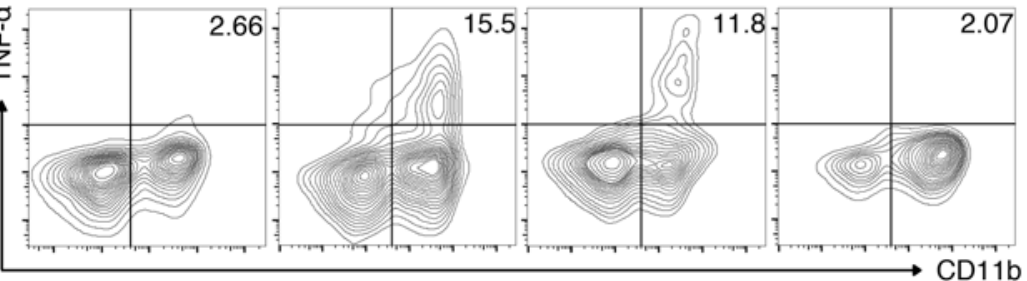

E Colon LPMC DW-PBS

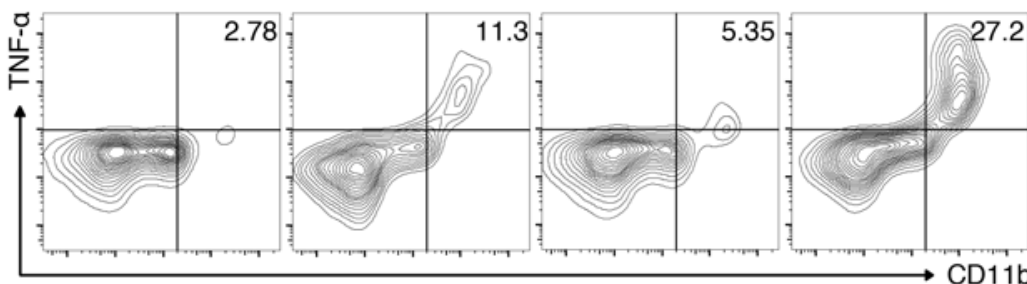

G Liver MC

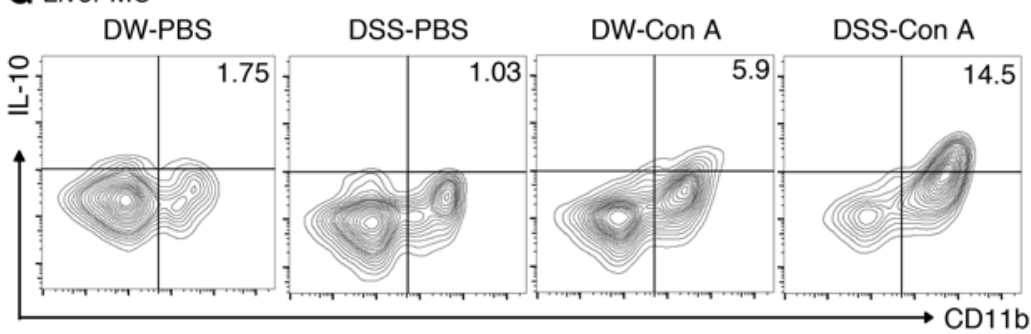

B

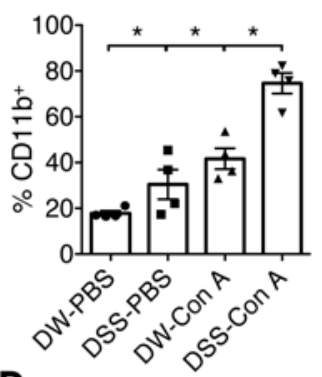

D

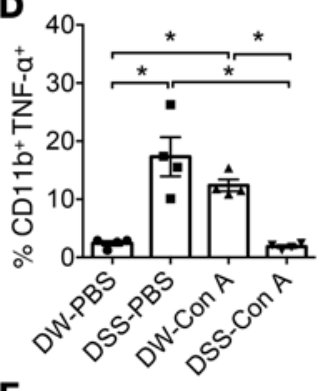

F
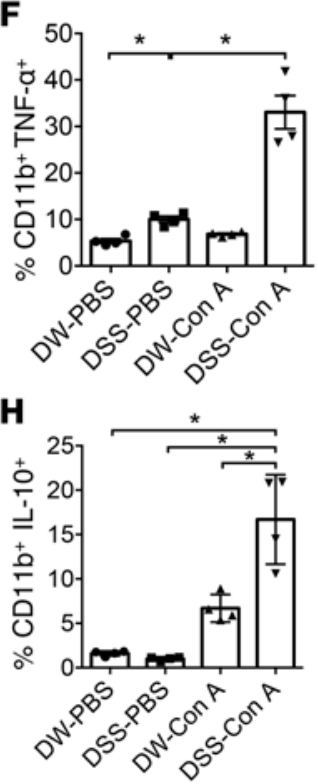

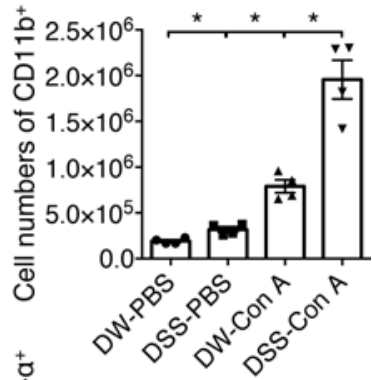

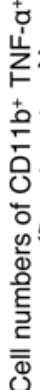
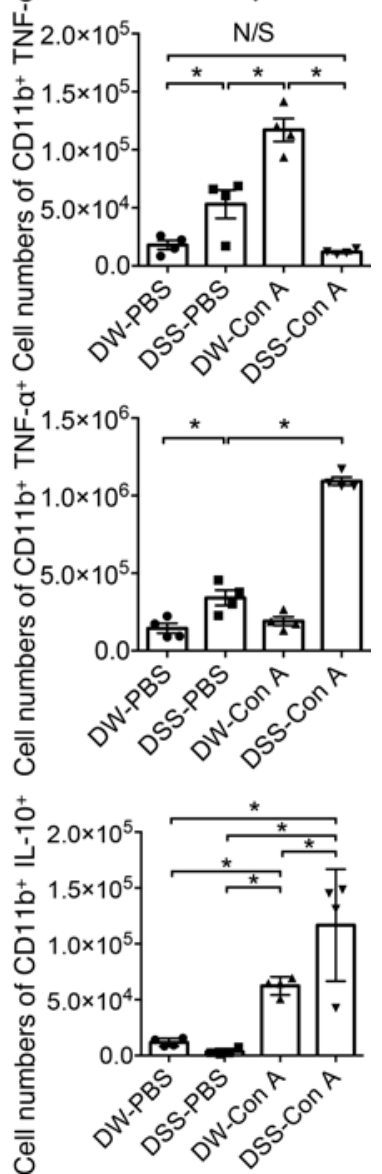

Figure 2. Liver DSS-Con A monocyte-derived macrophages display decreased TNF- $\alpha$ production and potentially increased IL-10 production upon LPS stimulation in vitro, and antigen-presenting potential in hepatic macrophages is reduced following DSS-Con A treatment. (A) Representative CD11b and CD11c staining of whole liver mononuclear cells (MCs) isolated from each experimental group. Each box and number indicates the population and the percentage of CD11 b+ cells in whole liver MCs. (B) Percentage (left) and number (right) of CD11 b+ cells in whole liver MCs of each experimental group ( $n=4 /$ group). (C) Representative surface CD11b and intracellular TNF- $\alpha$ staining of whole liver MCs isolated from each experimental group after 4 hours of LPS stimulation. Each number indicates the percentage resident in whole liver MCs. (D) Percentage and number of TNF- $\alpha^{+}$CD11b+ cells in the livers of mice from each experimental group ( $n=4$ /group). (E) Representative surface CD11b and intracellular TNF- $\alpha$ staining of whole lamina propria MCs isolated from each experimental group after 4 hours of LPS stimulation. Each number indicates the percentage resident in whole lamina propria MCs. (F) Percentage and number of TNF- $\alpha^{+}$CD11 $b^{+}$cells in the colon lamina propria of each experimental group ( $n=4$ /group). (C) Representative surface CD11b and intracellular IL-10 staining of whole liver MCs isolated from each experimental group after 4 hours of LPS stimulation. Each number indicates the percentage in whole liver MCs. (H) Percentage and number of IL-10+CD11 ${ }^{+}$cells in the livers of mice from each experimental group ( $n=4 /$ group). Data represent the mean \pm SEM. ${ }^{*} P$ $<0.05$ according to 1-way ANOVA with Tukey's multiple-comparison correction.

whereas colon lamina propria $\mathrm{CD}_{11} \mathrm{~b}^{+}$cells from DSS-Con A mice exhibited robust TNF- $\alpha$ production (Figure 2, E and F), clearly demonstrating the distinct phenotypes of the liver and colon cells. Moreover, we assessed antiinflammatory cytokine production by DSS-Con A macrophages upon LPS stimulation in vitro. Notably, DSS-Con A macrophages in the liver produced larger amounts of IL-10 following LPS stimulation in vitro (Figure 2, G and $\mathrm{H}$ ). The numbers of other immune-regulatory cell subsets, such as 
A

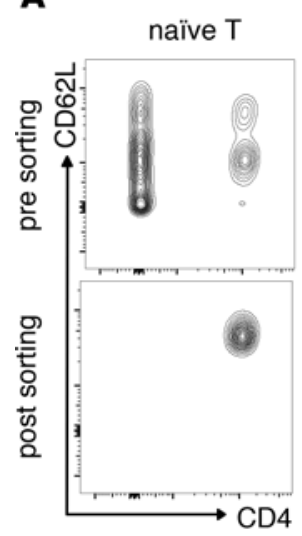

C

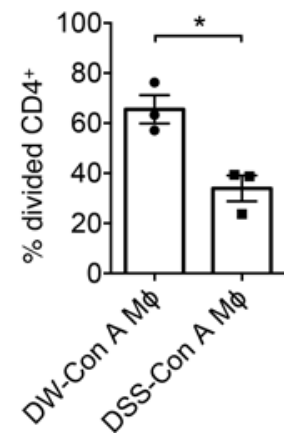

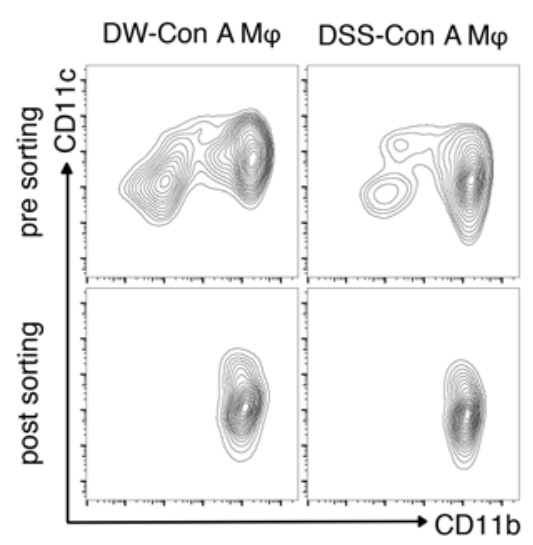

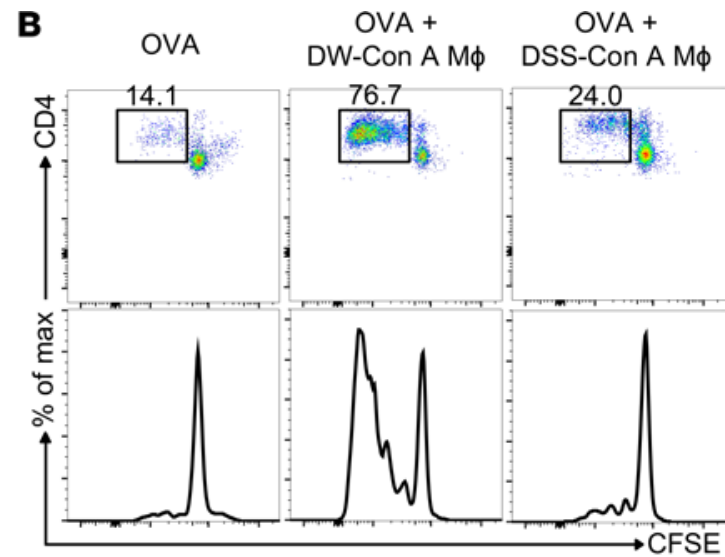

D

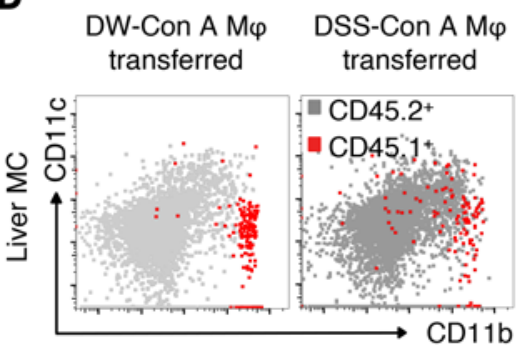

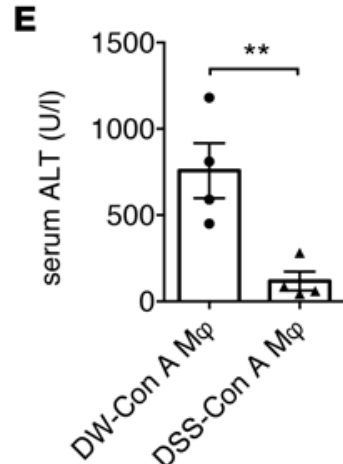

Figure 3. Liver DSS-Con A macrophages show impaired antigen-presenting function to $T$ cells in vitro and exert protective functions against excessive liver injury induced by Con A. (A) Gating strategy applied to sort CD4+CD62 $\mathrm{L}^{+}$cells from the spleens of OT-II mice and CD11 b+ cells from the livers of DSS-Con A-and DW-Con A-treated mice. (B) Proliferation of naive CFSE-labeled splenic CD4+ $T$ cells from OT-II mice and F4/80+CD11b+ cells from DSS-Con A- or DW-Con A-treated mice cocultured in the presence of ovalbumin. $C D 4^{+} T$ cells gated from $\mathrm{CD}^{+}{ }^{+} \mathrm{CD} 4^{+}$cells are shown. Each box and number indicates the population and percentage of proliferated cells. Data are representative of each experimental group. (C) Percentage of divided cells from each experimental group ( $n=3 /$ group). Data represent the mean \pm SEM. ${ }^{*} P<0.05$ according to a 2 -tailed Student's $t$ test. (D) To directly evaluate the role of adoptively transferred macrophages, recipient Ly5.2 mice were injected with clodronate liposome 24 hours before treatment and adoptively transferred with macrophages derived from DW-Con A- or DSS-Con A-treated Ly5.1 mice, followed by Con A $(20 \mathrm{mg} / \mathrm{kg})$ administration. Representative CD11b and CD11c staining of whole liver MCs isolated from each experimental group. Red dots indicate adoptively transferred CD45.1 ${ }^{+}$cells. (E) Serum ALT level in each experimental group ( $n=4 / \mathrm{group}$ ). Data represent the mean \pm SEM. ${ }^{* *} P<0.01$ according to a 2-tailed Student's $t$ test.

$\mathrm{CD} 11 \mathrm{c}^{+}$Siglec $\mathrm{H}^{+}$plasmacytoid DCs (pDCs) (19) and $\mathrm{CD} 4^{+} \mathrm{CD} 25^{+} \mathrm{Foxp} 3^{+}$Tregs (20), were decreased in the livers of DSS-Con A mice (Supplemental Figure 4, A-D).

Liver DSS-Con A macrophages show impaired antigen-presenting function to $T$ cells in vitro and exert protective functions against excessive liver injury induced by Con $A$. Next, we assessed the antigen-presenting function of hepatic $\mathrm{CD} 11 \mathrm{~b}^{+} \mathrm{F} 4 / 80^{+}$cells isolated from DSS-Con A-treated mice and compared it with the same function of $\mathrm{CD} 11 \mathrm{~b}^{+} \mathrm{F} 4 / 80^{+}$cells from DW-Con A-treated livers. DSS-Con A- or DW-Con Aderived $\mathrm{CD} 11 \mathrm{~b}^{+} \mathrm{F} 4 / 80^{+}$cells were cocultured with naive carboxyfluorescein succinimidyl ester-labeled (CFSE-labeled) $\mathrm{CD}^{+}{ }^{+} \mathrm{CD} 62 \mathrm{~L}^{+} \mathrm{T}$ cells derived from the spleens of OT-II mice in the presence of ovalbumin peptides (Figure $3 \mathrm{~A}$ ). After a 72-hour culture, naive $\mathrm{CD} 4^{+} \mathrm{T}$ cells extensively proliferated in the presence of DW-Con A-derived macrophages, whereas they exhibited less proliferation in the presence of DSS-Con A-derived macrophages (Figure 3, B and C). These data suggested that hepatic CD11 b macrophages derived from DSS-Con A-treated mice displayed an immune-regulatory phenotype that might contribute to the attenuation of acute liver injury. We then investigated whether DSS-Con A-derived $\mathrm{CD} 11 \mathrm{~b}^{+}$cells directly suppressed liver injury. $\mathrm{CD} 11 \mathrm{~b}^{+}$cells obtained from either DW-Con A-treated or DSS-Con A-treated Ly5.1+ WT mice were transferred to clodronate-pretreated Ly5.2+ mice to evaluate the role of adoptively transferred macrophages. We confirmed that $\mathrm{CD} 45.1^{+} \mathrm{CD} 11 \mathrm{~b}^{+}$cells substantially accumulated in the inflamed liver of the mice (Figure 3D). As shown in Figure 3E, transfer of DSS-Con $\mathrm{A}$-derived $\mathrm{CD} 11 \mathrm{~b}^{+}$cells significantly protected mice from subsequent Con A-induced liver injury. These 
A

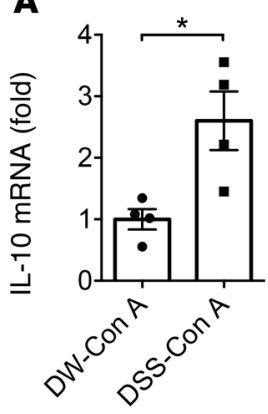

B

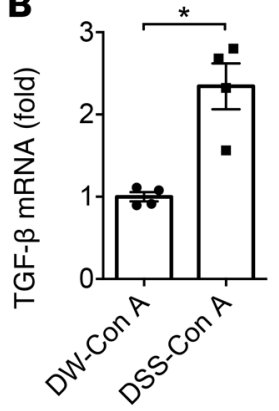

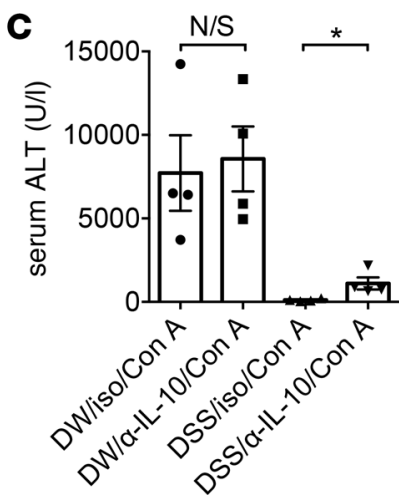

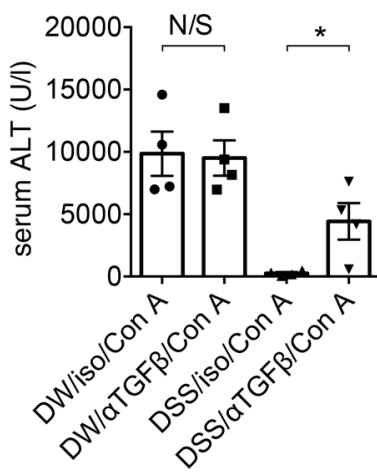

Figure 4. IL-10 and TGF- $\beta$ are involved in the attenuation of liver injury by tandem DSS-Con $\mathbf{A}$ administration. (A) IL-10 mRNA expression in whole liver cells ( $n=4$ /group). (B) TGF- $\beta$ mRNA expression in whole liver cells $\left(n=4 /\right.$ group). Data represent the mean \pm SEM. ${ }^{*} P<0.05$ according to a 2 -tailed Student's $t$ test. (C) Neutralizing anti-IL-10 and anti-TGF- $\beta$ monoclonal antibodies or control IgG was administrated 30 minutes before Con A treatment. Serum ALT level in each experimental group ( $n=4$ /group). Data represent the mean \pm SEM. ${ }^{*} P<0.05$ according to 1 -way ANOVA with Tukey's multiple-comparison correction.

results clearly demonstrated that $\mathrm{CD} 11 \mathrm{~b}^{+}$macrophages derived from DSS-Con A-treated mice were capable of attenuating Con A-induced acute liver injury. Activated Th1 cells play a critical role on the pathogenesis of Con A-induced acute liver injury, given that $\mathrm{Rag}^{\mathrm{I}^{--}}, \mathrm{Ifng}^{-/-}, \mathrm{Stat1}^{-/-}$, and $\mathrm{Tb} \times 2 \mathrm{1}^{-/-}$mice develop less severe liver injury (21-23). As expected, DSS-Con A-treated mice exhibited diminished accumulation of both $\mathrm{CD}^{+} \mathrm{CD}^{+}$and $\mathrm{CD}^{+} \mathrm{CD}^{+} \mathrm{T}$ cells in the liver (Supplemental Figure $5, \mathrm{~A}-\mathrm{C}$ ). To examine potential inflammatory cytokine production, liver MCs were stimulated with PMA/ionomycin for 4 hours in vitro, followed by intracellular IFN- $\gamma$ staining. Accordingly, as shown in Supplemental Figure 5, D-G, both $\mathrm{CD} 3{ }^{+} \mathrm{CD} 4^{+}$and $\mathrm{CD} 3^{+} \mathrm{CD} 8^{+} \mathrm{T}$ cells derived from DSS-Con A-treated livers produced less IFN- $\gamma$ than did DW-Con A mice. These data indicated that preexisting colitis with intestinal barrier degradation affected Con $\mathrm{A}$-induced $\mathrm{T}$ cell activation in the liver.

IL-10 and TGF- $\beta$ are involved in the attenuation of liver injury by tandem DSS-Con A administration. Given that the number of IL-10-producing macrophages increased in DSS-Con A-treated livers (Figure 2), we hypothesized that enhanced expression of regulatory cytokines promoted the attenuation of acute liver injury. Indeed, IL-10 and TGF- $\beta$ mRNA expression were significantly upregulated in whole livers derived from DSS-Con A-treated mice as compared with levels observed in DW-Con A-treated livers (Figure 4, $\mathrm{A}$ and $\mathrm{B})$. To investigate the direct role of IL-10 and TGF- $\beta$ in attenuating liver injury in this model, both DW- and DSS-administered mice were treated with an anti-IL-10 or anti-TGF- $\beta$ antibody 30 minutes prior to Con A administration. Neutralization of IL-10 and TGF- $\beta$ levels had little effect on Con A-induced liver injury, whereas neutralization of IL-10 and TGF- $\beta$ partially ablated the protective effect of DSS-Con A treatment on liver injury (Figure 4, C and D). These data suggested that IL-10 and TGF- $\beta$ were directly involved in the attenuation of liver injury by tandem DSS-Con A administration.

Continuous exposure to gut-derived products directly affect the susceptibility to subsequent liver injury. We then examined whether gut sterilization affects the severity of DSS-Con A-induced liver injury to clarify the involvement of gut microbiota in these mechanisms. Mice were orally administered a broad-spectrum antibiotic cocktail (ampicillin [Amp], vancomycin [VCM], neomycin [Neo], and metronidazole [MNZ]) 3 times weekly for 2 weeks, followed by DSS-Con A treatment (Figure 5A). We confirmed that both the severity of colitis and body weight loss were comparable between the control and gut-sterilized groups (Figure 5B). Notably, gut sterilization by the antibiotic cocktail followed by DSS-Con A treatment ablated the protective effect against liver injury, as measured by serum alanine aminotransferase (ALT) levels and compared with control mice without gut sterilization (Figure 5C), suggesting that gut microbiota play a substantial role in the protective mechanism. Importantly, pretreatment with Neo, which mainly depletes gram-negative bacteria, followed by DSS-Con A treatment worsened colitis symptoms but did not ablate the protective effect against Con A-induced liver injury, as compared with that observed in control mice without gut sterilization (Figure 5, B and C). These results suggested that increased exposure to microbial products (especially gram-positive microbiota) accompanied by intestinal barrier disruption played a substantial role in the induction of immune tolerance in the liver. We further determined whether the TLR/ 
A

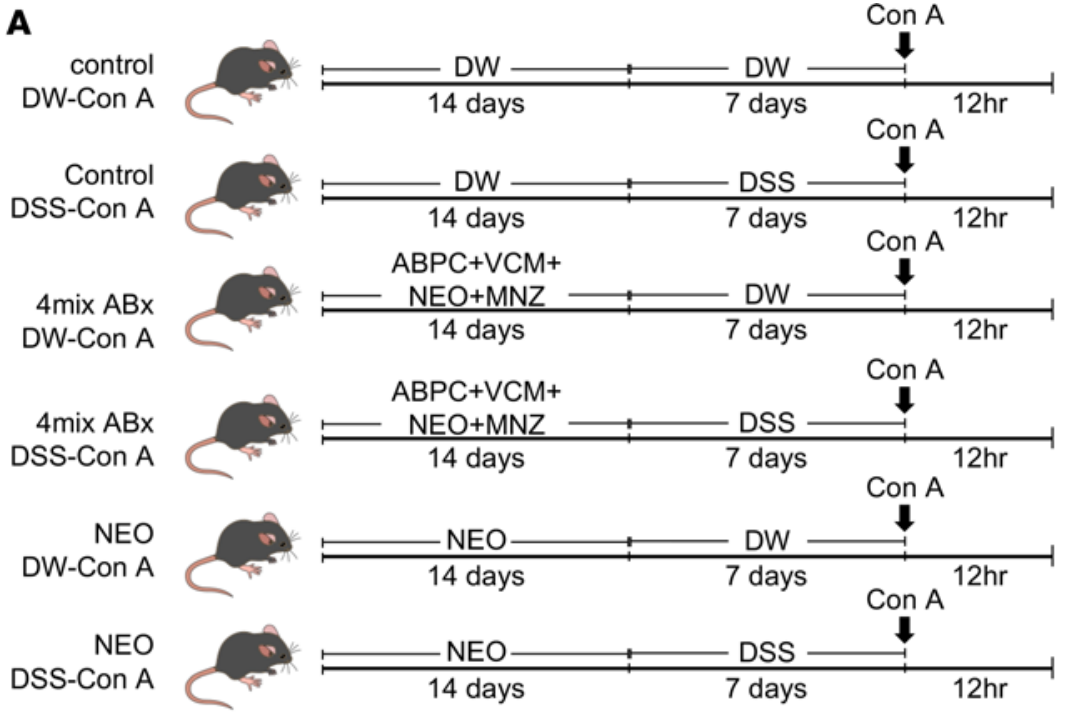

B
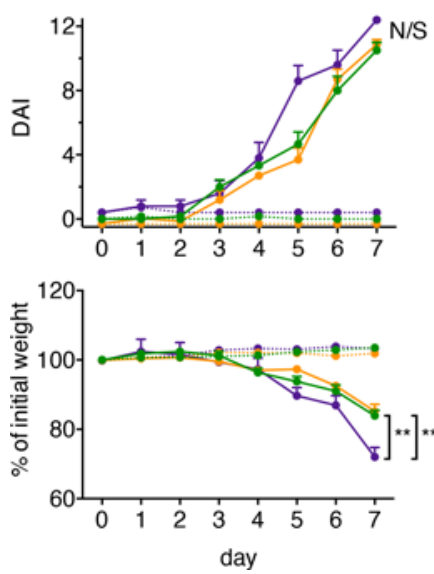

- Control DW-Con A

DSS-Con

DSS-Con A

DW-Con A

$\mathrm{ABPC}+\mathrm{VCM}+\mathrm{NEO}+\mathrm{MNZ}$

DSS-Con A

- NEO

DW-Con A

- NEO

DSS-Con A
C

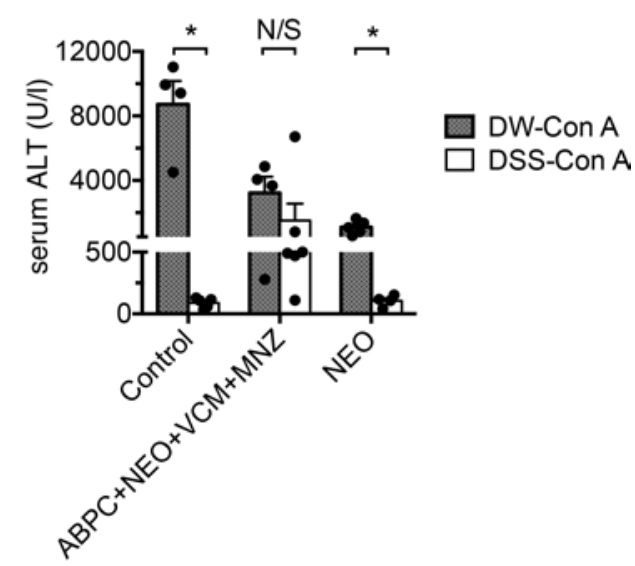

Figure 5. Continuous exposure to gut-derived products directly affects the susceptibility to subsequent liver injury. (A) Antibiotic cocktails or single antibiotics were orally administrated 6 times over a 2-week period, followed by DSS-Con A treatment. (B) Body weight and DAI on days 0-7 of mice in each group ( $n=6 /$ group). Data represent the mean $\pm \mathrm{SEM}$. ${ }^{* *} P<0.01$ according to 1-way ANOVA with Tukey's multiple-comparison correction. (C) Serum ALT level in mice from each experimental group ( $n=4-6 /$ group). Data represent the mean \pm SEM. ${ }^{*} P<0.05$ according to a 2 -tailed Student's $t$ test. 
A
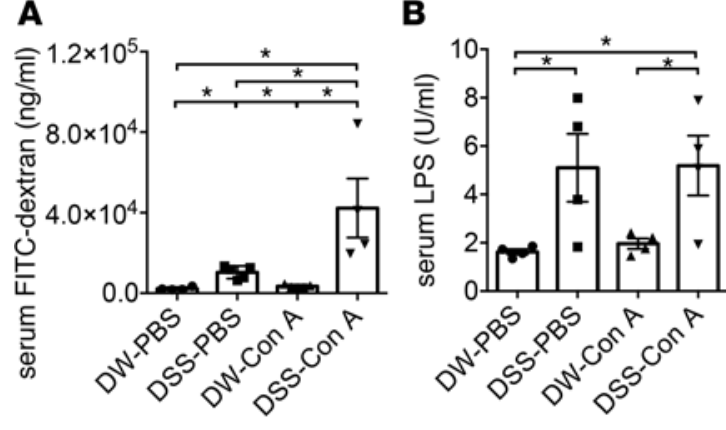

C

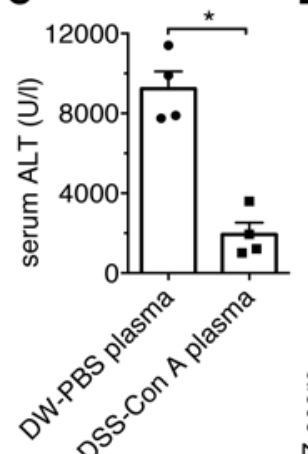

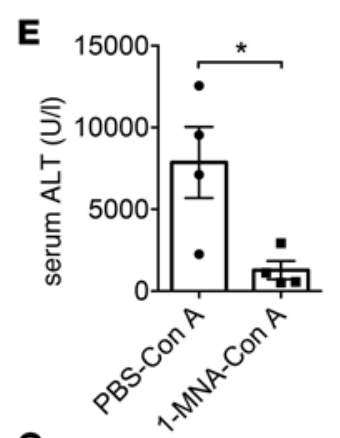

G
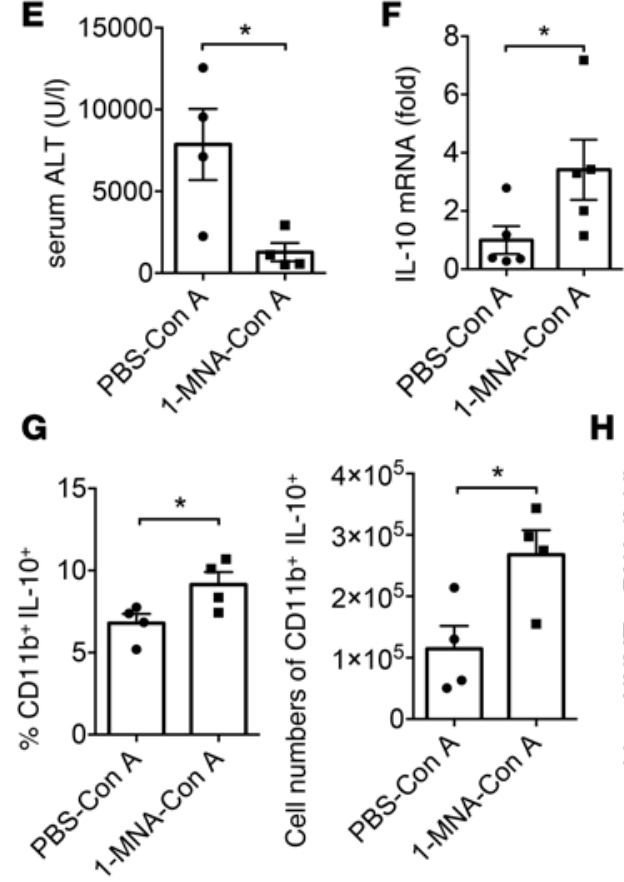

(2)
H
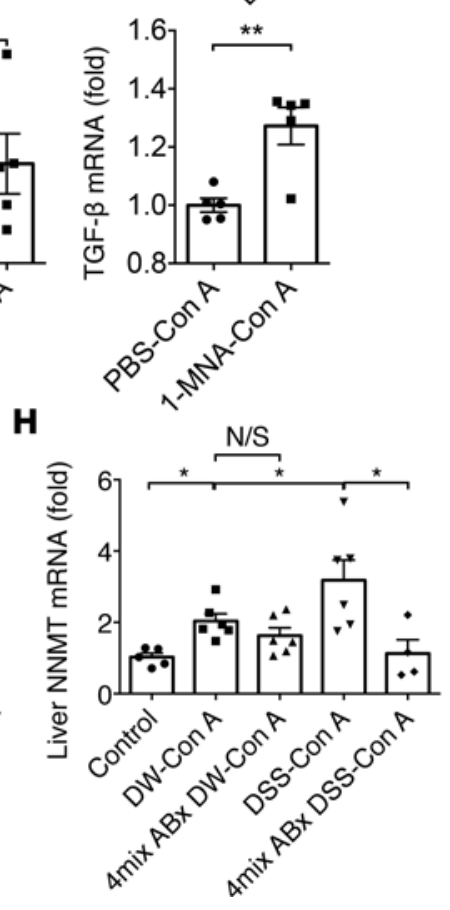

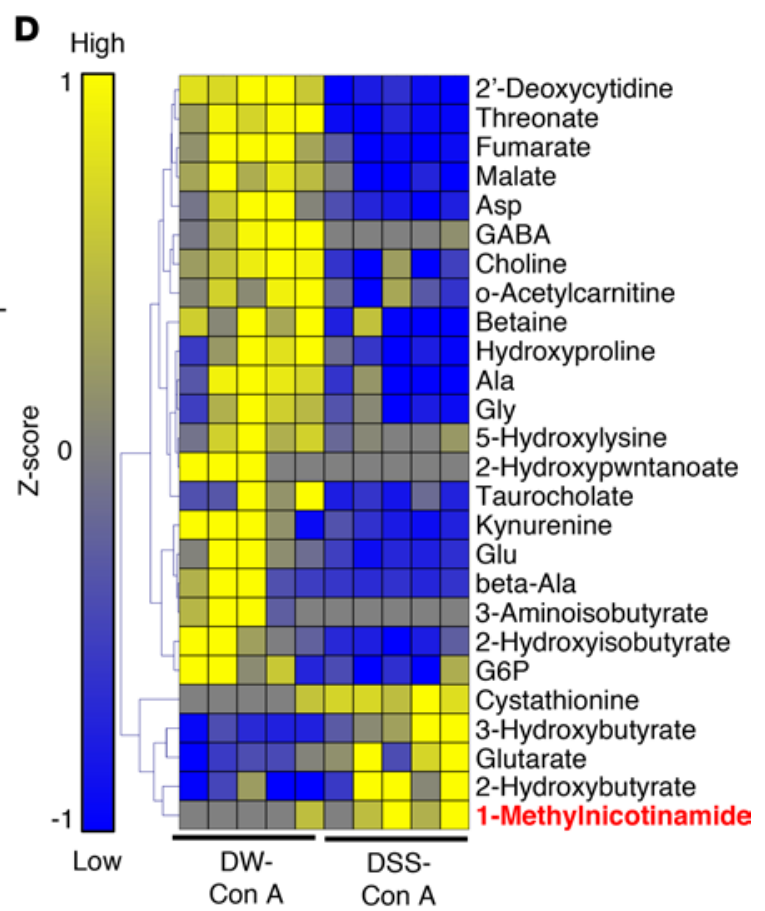

Figure 6. Nicotinamide $\mathrm{N}$-methyltransferase (NNMT) upregulation and concomitant increase in 1-methylnicotinamide (1-MNA) levels play integral roles in the induction of immune tolerance via the gut-liver axis. (A) Serum FITC-dextran levels 4 hours after gavage administration into mice in each experimental group ( $n=4-5 /$ group). (B) Serum LPS level of each experimental group ( $n=4 /$ group). (C) Mice received plasma derived from distilled water-PBStreated (DW-PBS-treated) or dextran sulfate sodium DSS-Con A-treated mice i.v. followed by Con A administration, and serum alanine aminotransferase (ALT) was measured in mice from each experimental group ( $n=4 /$ group). (D) CE-TOFMS-based metabolome analysis of plasma. Hierarchical clustering and heatmap analysis of the identified metabolites exhibiting significantly different levels in DW-Con A- and DSS-Con A-treated mice. (E) 1-MNA (100 mg/ $\mathrm{kg}$ ) or PBS was i.v. administered 15 minutes before Con A treatment, and serum ALT was measured in mice from each experimental group ( $n=4 / \mathrm{group}$ ). (F) IL-10 and TGF- $\beta$ mRNA expression in whole liver cells of mice from each experimental group ( $n=5 /$ group). (C) Percentage and number of CD11b+IL-10+ cells in the livers of mice from each experimental group ( $n=4$ /group). Data represent means \pm SEM. ${ }^{*} P<0.05$ according to a 2-tailed Student's $t$ test. (H) NNMT mRNA expression in whole liver cells ( $n=4$ /group) of mice from each experimental group. Data represent means $\pm S E M$. ${ }^{*} P<0.05$ according to 1-way ANOVA with Tukey's multiple-comparison correction.

ed mice (Supplemental Figure 7A). Consistently, DSS-Con A-derived plasma induced higher IL-10 production from liver $\mathrm{CD} 11 \mathrm{~b}^{+}$cells as compared with DW-PBS-derived plasma (Supplemental Figure 7B). These data suggested that molecules in plasma from DSS-Con A-treated mice exhibited a protective effect against Con A-induced liver injury; therefore, we determined the metabolites present in plasma derived from DSS-Con A-treated mice by capillary electrophoresis TOF mass spectrometrybased (CE-TOFMS-based) metabolome analysis (Figure 6D). Several metabolites in plasma derived from DSS-Con A-treated mice showed higher levels relative to those in plasma derived from DWCon A-treated mice, including 1-MNA, a terminal product of nicotinamide (NAM) clearance. We then confirmed that 1-MNA administration protected mice against Con A-induced liver injury, along with increased mRNA expression of TGF- $\beta$ and IL-10, and especially induction of IL-10-producing macrophages in the liver (Figure 6, E-G). Moreover, the mRNA expression of NNMT, which catalyz- 


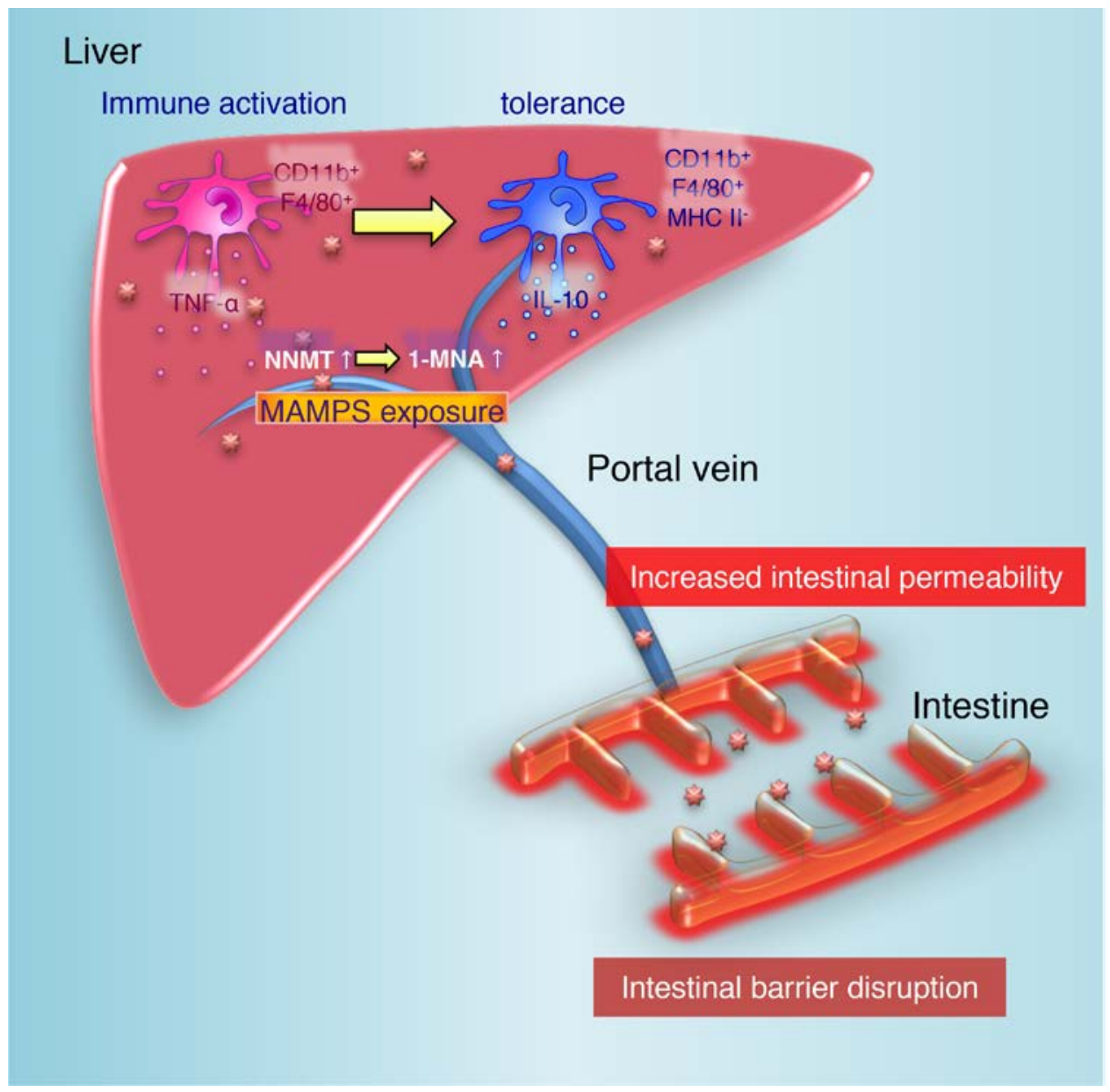

Figure 7. Proposed mechanism for the induction of immune tolerance in livers with a disrupted intestinal barrier. The liver is exposed to gut-derived antigens through the portal vein. In steady state conditions, hepatic inflammatory macrophages play a critical role in their elimination. In the presence of a disrupted intestinal barrier, continuous exposure to microbe-associated molecular patterns increases NNMT expression, along with a concomitant increase in 1-MNA levels, and shifts hepatic inflammatory macrophages to a regulatory phenotype accompanied by potential IL-10 production in response to further stimulation to avoid excessive tissue damage. MAMPS, microbe-associated molecular patterns.

es NAM methylation to produce 1-MNA, was significantly higher in DSS-Con A-treated livers than in control mice. Finally, gut sterilization via the antibiotic cocktail followed by DSS-Con A treatment ablated NNMT upregulation in the liver (Figure $6 \mathrm{H}$ ). These results collectively suggest that NNMT upregulation by exposure to gut-derived products and concomitant increases in 1-MNA play an integral role in the induction of immune tolerance via the gut-liver axis.

\section{Discussion}

The gut-liver axis is of clinical importance as a potential therapeutic target in a wide range of liver diseases, including nonalcoholic steatohepatitis (NASH) (24), alcoholic liver diseases (25), and PSC (7). Recent reports have suggested that altered gut microbiota and increased intestinal permeability contribute to liver disease initiation and progression (26-28). The present study revealed an unexpected role of the gut-liver axis in maintaining immunological balance in the liver. Herein, we demonstrated that a distinctive state with intestinal barrier disruption and continuous exposure to microbial products protected mice from further liver injury, along with the emergence of immunosuppressive macrophages. As a proposed mechanism, induction of immunological tolerance in the liver was IL-10 and TGF- $\beta$ dependent, as the protective effect against DSS-Con A administration was partially canceled by treatment with anti-IL-10 or antiTGF- $\beta$ antibodies. Furthermore, certain gut microbiota or microbial products played a substantial role via the gut-liver axis based on the attenuated protective effect against DSS-Con A administration by gut sterilization. We identified 1-MNA as a candidate metabolite capable of directly suppressing liver injury by inducing IL-10-producing macrophages in the liver (Figure 7).

We previously reported that an anti-Fas-induced fulminant-hepatitis model exhibited a deteriorated response to DSS-induced colitis (13). Similar liver inflammation linked with increased intestinal permeability and dysbiosis has been observed both in human diseases and murine models, including those related to jejunal self-filling blind loops (29) and alcoholic and nonalcoholic liver diseases. Con- 
trary to these previous findings, we demonstrated that DSS-colitis pretreatment protected mice from immune-mediated hepatitis initiated by Con A. It is worth mentioning that the severity of intestinal inflammation is conversely correlated with subsequent liver injury. Specific subsets of innate immune cells in the liver adequately respond to gut-derived antigens, whereas excessive antigen stimuli induce immune permissiveness to avoid further liver injury $(3,30)$. For example, LPS induces strong immune responses in a variety of cells, whereas subsequent challenge - even with a high dose of LPS — induces increased production of antiinflammatory cytokines, a state widely known as endotoxin tolerance (18). On the other hand, pretreatment with an extremely low dose of endotoxin has an opposite effect in that it potentiates a proinflammatory response to subsequent endotoxin challenge, referred to as the Shwartzman-like reaction (31). It is generally believed that the initial magnitude of the immune reaction determines the immunological fate, namely immune priming or immune tolerance (32). This 2-pronged phenomenon might account for the discrepancy between our findings and those from previous reports illustrating that continuous mild intestinal inflammation induced by chronic DSS administration enhanced hepatic inflammation and fibrogenesis in an experimental NASH model (33).

Whether the mechanism behind the liver tolerance demonstrated in the present study is distinct from LPS tolerance is an important issue. First, the expression of IRAK3, a molecule contributing to LPS tolerance (34), was not induced but was decreased in the livers of DSS-Con A-treated mice. Second, we observed equally suppressed liver inflammation in MyD88-deficient mice administered DSS-Con A (Supplemental Figure 6C). Third, pretreatment with Neo to examine the role of gram-negative bacteria failed to cancel the induction of immune tolerance in DSS-Con A-treated mice. These findings collectively suggest that immune tolerance in this model was independent of LPS tolerance.

We recently reported the critical role of TNF- $\alpha$-producing CCR9+CD $11 b^{+}$macrophages in the pathogenesis of Con A-induced hepatitis $(16,17)$. These macrophages are characterized as classically activated macrophages that contribute to host defenses against a variety of bacteria and viruses and play a substantial role in antitumor immunity. However, in the present study, we found that continuous exposure to gut-derived antigens with diminished intestinal integrity induced immunological tolerance in the liver, along with the emergence of IL-10-producing macrophages, and directly protected mice from Con A-induced acute liver injury. It is reasonable that recruited hepatic macrophages shifted from inflammatory to regulatory functions in the hepatic microenvironment continuously exposed to microbial products through the portal vein, rather than being differentially recruited directly to the liver as regulatory macrophages. Accordingly, Con A-pretreated mice (a state of severe hepatitis) were not protected from subsequent DSS-induced colitis, suggesting that the emergence of immune-suppressive macrophages via the gut-liver axis was unique to the liver. As candidate products potentially contributing to the induction of immune tolerance in the liver, we confirmed that 1-MNA directly contributed to the attenuation of Con A-induced liver injury by inducing IL-10-producing macrophages. Recent studies have suggested that 1-MNA possesses biological activity and is not merely a terminal product of NAM clearance. 1-MNA is methylated from NAM by NNMT, which is specifically expressed in hepatocytes (35), and its expression in the liver is associated with serum cholesterol, triglycerides, and other metabolic parameters (36). Mechanistically, increasing NNMT expression or 1-MNA levels stabilizes the sirtuin 1 protein, an effect that is required for its metabolic benefits (36). Increased NNMT expression and 1-MNA release have been reported in humans and rodent models of inflammation, including those involving Con Ainduced acute liver injury and pulmonary hypertension (37-39), indicating a protective role for NNMT in compensating for inflammation. We demonstrated that increased NNMT expression in the liver following DSS-Con A administration was abrogated by gut sterilization, suggesting potential regulation of NNMT expression in the liver by gut microbes.

In summary, our findings showed that $\mathrm{IL}-10$-producing $\mathrm{CD} 11 \mathrm{~b}^{+}$macrophages contribute to immune tolerance in the inflamed liver under intestinal inflammation conditions accompanied by diminished intestinal barrier function. Our results provided a potentially novel mechanism of immune tolerance in the liver via the gut-liver axis, as well as insight into a therapeutic strategy for treating gut-related liver diseases.

\section{Methods}

Animals. WT C57BL/6J mice were purchased from Japan CLEA. Mice were maintained under specific pathogen-free (SPF) conditions in the Animal Care Facility of Keio University School of Medicine. Experiments were performed with age- and sex-matched mice at 8-12 weeks of age. 
Experimental protocols of Con A-induced hepatitis. Con A (type IV) was purchased from MilliporeSigma. Con A ( $15 \mathrm{mg} / \mathrm{kg})$ were i.v. administered into the tail vein of mice 12 hours before liver resection.

Experimental protocols of DSS-induced colitis as model of mucosal barrier degradation. DSS (molecular weight $36-50 \mathrm{kDa}$ ) was purchased from MP Biomedicals. To induce mucosal barrier degradation by acute experimental colitis, mice received 1.5\%-2.0 \% DSS dissolved in sterile DW ad libitum for 4-7 days.

Assessment of clinical activity in DSS-treated mice. Assessment of clinical activity in DSS-treated mice were evaluated as described previously (40). Mice were weighed daily and visually inspected for diarrhea and rectal bleeding. DAI was assessed in a blinded fashion. No weight loss was registered as 0 , weight loss of $1 \%-5 \%$ from baseline was assigned 1 point, with $6 \%-10 \%$ as 2 points, $11 \%-20 \%$ as 3 points, and $>20 \%$ as 4 points. For stool consistency, 0 points were assigned for well-formed pellets, 2 points for pasty and semiformed stools that did not adhere to the anus, and 4 points for liquid stools that adhered to the anus. For bleeding, 0 was assigned for no blood, 2 points for positive bleeding, and 4 points for gross bleeding.

Preparation of liver MCs and intestinal LPMCs. Liver MCs were separated from the liver as described previously (16). Single-cell suspensions of intestinal LPMCs were prepared as previously described (41), with slight modifications. Details are described in the Supplemental Methods.

Flow cytometry. After blocking with anti-FcR (CD16/32, 2.4G2, BD Pharmingen) for 20 minutes, the cells were incubated with the specific fluorescence-labeled mAbs used for surface staining at $4^{\circ} \mathrm{C}$ for 30 minutes, followed by permeabilization and intracellular staining in some cases. Antimouse mAbs used are listed in the Supplemental Methods.

Quantitative PCR ( $q P C R$ ). All qPCR assays were performed as described previously (16). Details and the predesigned primers are described in the Supplemental Methods.

Macrophage depletion. To deplete macrophages, mice were i.v. injected Clodronate Liposome $(200 \mu \mathrm{g} /$ body; FormuMax) 24 hours before Con A administration.

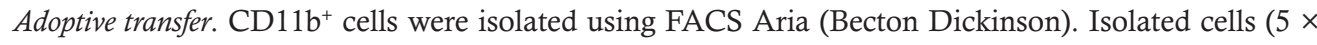
$10^{5}$ cells) transferred immediately injected with Con A.

Neutralizing IL-10 or TGF- $\beta$. To neutralize IL-10 or TGF- $\beta$, mice were treated with neutralizing antibodies to IL-10 (JES5-2A5; $200 \mu \mathrm{g} /$ body, eBioscience) or TGF- $\beta$ (1D11; $100 \mu \mathrm{g} /$ body, Gene Tex) or isotype-matched antibodies 30 minutes before Con A administration.

Evaluation of intestinal permeability. Mice were orally provided with 4,000 Da FITC dextran (MilliporeSigma) via nasogastric tube after being fasted for 6 hours. Blood samples were collected from the tail vein at 4 hours. The concentration of FITC from obtained serum was measured by spectrophoto fluorometry according to the instructions. Serum from mice not administered with FITC-dextran is used to determine the background.

Gut sterilization with broad-spectrum antibiotics. Mice were treated with broad-spectrum antibiotics via nasogastric tube ( $500 \mu 1 /$ each) 3 times a week for 2 weeks. The following amount of antibiotics were used; Amp (6.7 g/1), Neo (6.7 g/1), VCM (3.3 g/1), and MNZ (6.7 g/1).

Pretreatment with plasma derived from portal vein injection in vivo. Mice were i.v. administered with plasma derived from portal vein $(200 \mu 1$ /body) derived from DW-PBS or DSS-Con A mice at 2 hours before Con A injection.

CE-TOFMS-based metabolome analysis. A quantitative analysis of charged metabolites by CE-TOFMS was performed as described previously(42). Details are described in the Supplemental Methods.

Statistics. Results are expressed as mean \pm SEM. Data groups were analyzed with GraphPad Prism software using 1-way ANOVA with Tukey's multiple-comparison correction or 2-tailed Student's $t$ test. A $P$ value less than 0.05 was considered statistically significant.

Study approval. All animal protocols described in this study were performed in accordance with Institutional Guidelines on Animal Experimentation at Keio University and were approved by The Keio University IACUC.

\section{Author contributions}

NT helped design the study, performed experiments, and analyzed the data; NN conceived and designed the study, analyzed the data, and wrote the paper; PSC, TA, T. Teratani, TS, AY, SS, RM, and T. Katayama performed experiments; T. Tsukimi and SF performed metabolome analysis; YM and HE helped design the study; and T. Kanai helped conceive and supervise the study. 


\section{Acknowledgments}

We thank A. Hayashi, S. Chiba, K. Miyamoto, and Y. Koda (Keio University School of Medicine) for technical assistance and valuable suggestions. We would like to thank Editage (www.editage.jp) for English language editing. This study was supported in part by grants-in-aid for scientific research, scientific research on priority areas, exploratory research and creative scientific research from the Japanese Ministry of Education, culture, sports, science, and technology; the Japanese Ministry of Health, Labor, and Welfare; the Japan Medical Association; the Foundation for Advancement of International Science; JST PRESTO (JPMJPR1537 to SF); and Keio University Medical Fund.

Address correspondence to: Nobuhiro Nakamoto or Takanori Kanai, Division of Gastroenterology and Hepatology, Department of Internal Medicine, Keio University School of Medicine, Tokyo 160-8582, Japan. Phone: 81.3.3353.1211; Email: nobuhiro@z2.keio.jp (N. Nakamoto); takagast@z2.keio.jp (T. Kanai).

1. Crispe IN. The liver as a lymphoid organ. Annu Rev Immunol. 2009;27:147-163.

2. Nakamoto N, Kanai T. Role of toll-like receptors in immune activation and tolerance in the liver. Front Immunol. 2014;5:221

3. Thomson AW, Knolle PA. Antigen-presenting cell function in the tolerogenic liver environment. Nat Rev Immunol. 2010;10(11):753-766.

4. Balmer ML, et al. The liver may act as a firewall mediating mutualism between the host and its gut commensal microbiota. $S c i$ Transl Med. 2014;6(237):237ra66.

5. Calne RY, et al. Induction of immunological tolerance by porcine liver allografts. Nature. 1969;223(5205):472-476

6. Bertolino P, Trescol-Biémont MC, Rabourdin-Combe C. Hepatocytes induce functional activation of naive CD8+ T lymphocytes but fail to promote survival. Eur J Immunol. 1998;28(1):221-236.

7. Tabibian JH, Talwalkar JA, Lindor KD. Role of the microbiota and antibiotics in primary sclerosing cholangitis. Biomed Res Int. 2013;2013:389537.

8. Celaj S, et al. The microbiota regulates susceptibility to Fas-mediated acute hepatic injury. Lab Invest. 2014;94(9):938-949.

9. Mazagova M, et al. Commensal microbiota is hepatoprotective and prevents liver fibrosis in mice. FASEB J. 2015;29(3):1043-1055.

10. Nakamoto N, et al. Commensal Lactobacillus Controls Immune Tolerance during Acute Liver Injury in Mice. Cell Rep. 2017;21(5):1215-1226.

11. Lazaridis KN, LaRusso NF. Primary Sclerosing Cholangitis. N Engl J Med. 2016;375(12):1161-1170.

12. Eksteen B. The Gut-Liver Axis in Primary Sclerosing Cholangitis. Clin Liver Dis. 2016;20(1):1-14.

13. Mikami Y, et al. Macrophages and dendritic cells emerge in the liver during intestinal inflammation and predispose the liver to inflammation. PLoS One. 2014;9(1):e84619.

14. Krenkel O, Tacke F. Liver macrophages in tissue homeostasis and disease. Nat Rev Immunol. 2017;17(5):306-321.

15. Scott CL, et al. Bone marrow-derived monocytes give rise to self-renewing and fully differentiated Kupffer cells. Nat Commun. 2016;7:10321

16. Nakamoto N, et al. CCR9+ macrophages are required for acute liver inflammation in mouse models of hepatitis. Gastroenterology. 2012;142(2):366-376.

17. Amiya T, et al. Bone marrow-derived macrophages distinct from tissue-resident macrophages play a pivotal role in Concanavalin A-induced murine liver injury via CCR9 axis. Sci Rep. 2016;6:35146.

18. Biswas SK, Lopez-Collazo E. Endotoxin tolerance: new mechanisms, molecules and clinical significance. Trends Immunol. 2009;30(10):475-487.

19. Zhang J, et al. Characterization of Siglec-H as a novel endocytic receptor expressed on murine plasmacytoid dendritic cell precursors. Blood. 2006;107(9):3600-3608.

20. Rudensky AY. Regulatory T cells and Foxp3. Immunol Rev. 2011;241(1):260-268.

21. Tiegs G, Hentschel J, Wendel A. A T cell-dependent experimental liver injury in mice inducible by concanavalin A. J Clin Invest. 1992;90(1):196-203.

22. Tagawa Y, Sekikawa K, Iwakura Y. Suppression of concanavalin A-induced hepatitis in IFN-gamma(-/-) mice, but not in TNF-alpha(-/-) mice: role for IFN-gamma in activating apoptosis of hepatocytes. J Immunol. 1997;159(3):1418-1428.

23. Siebler J, et al. A key pathogenic role for the STAT1/T-bet signaling pathway in T-cell-mediated liver inflammation. Hepatology. 2003;38(6):1573-1580.

24. Zhu L, et al. Characterization of gut microbiomes in nonalcoholic steatohepatitis (NASH) patients: a connection between endogenous alcohol and NASH. Hepatology. 2013;57(2):601-609.

25. Yan AW, et al. Enteric dysbiosis associated with a mouse model of alcoholic liver disease. Hepatology. 2011;53(1):96-105.

26. Yoshimoto S, et al. Obesity-induced gut microbial metabolite promotes liver cancer through senescence secretome. Nature. 2013;499(7456):97-101.

27. Sabino J, et al. Primary sclerosing cholangitis is characterised by intestinal dysbiosis independent from IBD. Gut. 2016;65(10):1681-1689.

28. Iwasawa $\mathrm{K}$, et al. Characterisation of the faecal microbiota in Japanese patients with paediatric-onset primary sclerosing cholangitis. Gut. 2017;66(7):1344-1346.

29. Goulet O, Joly F. [Intestinal microbiota in short bowel syndrome]. Gastroenterol Clin Biol. 2010;34 Suppl 1:S37-S43.

30. Crispe IN. Immune tolerance in liver disease. Hepatology. 2014;60(6):2109-2117. 
31. Heremans H, Van Damme J, Dillen C, Dijkmans R, Billiau A. Interferon gamma, a mediator of lethal lipopolysaccharide-induced Shwartzman-like shock reactions in mice. J Exp Med. 1990;171(6):1853-1869.

32. Morris MC, Gilliam EA, Li L. Innate immune programing by endotoxin and its pathological consequences. Front Immunol. 2014;5:680

33. Gäbele E, et al. DSS induced colitis increases portal LPS levels and enhances hepatic inflammation and fibrogenesis in experimental NASH. J Hepatol. 2011;55(6):1391-1399.

34. Kobayashi K, Hernandez LD, Galán JE, Janeway CA, Medzhitov R, Flavell RA. IRAK-M is a negative regulator of Toll-like receptor signaling. Cell. 2002;110(2):191-202.

35. Aksoy S, Szumlanski CL, Weinshilboum RM. Human liver nicotinamide N-methyltransferase. cDNA cloning, expression, and biochemical characterization. J Biol Chem. 1994;269(20):14835-14840.

36. Hong S, et al. Nicotinamide N-methyltransferase regulates hepatic nutrient metabolism through Sirt1 protein stabilization. Nat Med. 2015;21(8):887-894.

37. Jakubowski A, Sternak M, Jablonski K, Ciszek-Lenda M, Marcinkiewicz J, Chlopicki S. 1-Methylnicotinamide protects against liver injury induced by concanavalin A via a prostacyclin-dependent mechanism: A possible involvement of IL- 4 and TNF- $\alpha$. Int Immunopharmacol. 2016;31:98-104.

38. Kim HC, et al. Expression and functional significance of nicotinamide N-methyl transferase in skeletal muscles of patients with chronic obstructive pulmonary disease. Am J Respir Crit Care Med. 2010;181(8):797-805.

39. Sternak M, et al. Nicotinamide N-methyltransferase (NNMT) and 1-methylnicotinamide (MNA) in experimental hepatitis induced by concanavalin A in the mouse. Pharmacol Rep. 2010;62(3):483-493.

40. Hayashi A, et al. A single strain of Clostridium butyricum induces intestinal IL-10-producing macrophages to suppress acute experimental colitis in mice. Cell Host Microbe. 2013;13(6):711-722.

41. Mikami Y, et al. Competition between colitogenic Th1 and Th17 cells contributes to the amelioration of colitis. Eur J Immunol. 2010;40(9):2409-2422.

42. Mishima E, et al. Alteration of the Intestinal Environment by Lubiprostone Is Associated with Amelioration of Adenine-Induced CKD. J Am Soc Nephrol. 2015;26(8):1787-1794 